\title{
Gender and Peripheral Arterial Disease
}

\author{
Tracie C. Collins, MD, MPH, Maria Suarez-Almazor, MD, PhD, Ruth L. Bush, MD, \\ and Nancy J. Petersen, PhD
}

Objective: The aim of this study is to determine gender differences in the risk factor profile and leg symptoms of peripheral arterial disease (PAD).

Methods: We identified men and women with PAD from a cohort of patients within a primary care clinic setting. We screened patients 50 years of age and older. We diagnosed PAD based on an anklebrachial index (ABI) level of less than 0.9 ; the $\mathrm{ABI}$ is the ratio of ankle and arm systolic blood pressure measurements. Patients completed 4 questionnaires, one of which was used to ascertain leg symptoms related to compromised blood flow, the San Diego Claudication Questionnaire (SDCQ). Additional questionnaires were used to determine the patient's medical history, walking impairment, and health-related quality of life.

Results: We enrolled 403 patients stratified by race and gender including 55 white women, 82 African American women, and 71 Hispanic women. There were no significant differences by gender in the prevalence of disease. The prevalence of PAD was $9.1 \%$ in white women, $21.9 \%$ in African American women, and 14.1\% in Hispanic women $(P=.11)$. Risk factors for PAD were the same for women and men (ie, diabetes mellitus, current smoking, and use of blood pressure medication). Walking impairment subscale scores were lower for women with PAD when compared with women without PAD and to men with disease. Scores for physical function and general health were lower for women versus men with PAD.

Conclusions: The prevalence of PAD, a common disease within primary care clinics, does not vary by gender. Women with PAD are at greater risk for a compromise in daily function and quality of life. Future research is needed to prevent walking impairment and improve limb functioning in patients, particularly women, with PAD. (J Am Board Fam Med 2006;19:132-40.)

\section{Case Illustration}

A 63-year-old African American woman with leg discomfort comes into your practice setting complaining of cramping in legs when she walks. Retired for 8 years, she goes walking at a shopping mall with a friend 2 times per week but she must stop several times and she is unable to keep up with

Submitted 26 May 2005; revised 20 July 2005; accepted 26 July 2005.

From the Houston Center for Quality of Care and Utilization Studies, Michael E. DeBakey Veterans Affairs Medical Center, and Section of Health Services Research, Baylor College of Medicine, Houston, TX (TCC, MS-A, NJP); and Michael E. DeBakey Department of Surgery, Division of Vascular Surgery and Endovascular Therapy, Baylor College of Medicine, Houston, TX (RLB).

Funding: This work was supported in part by resources from the Robert Wood Johnson Foundation and the use of facilities at the Houston Center for Quality of Care and Utilization Studies, Michael E. DeBakey Veterans Affairs Medical Center. TCC is a recipient of an Advanced Clinical Research Career Development Award from the Department of Veterans Affairs (CRCD 735A).

Conflict of interest: none declared.

Corresponding author: Tracie Collins, MD, MPH, Department of Veterans Affairs, 2002 Holcombe Boulevard (152), Houston, TX 77030 (E-mail: tcollins@bcm.tmc.edu). her friend. Her leg symptoms resolve promptly with rest, and she has no rest pain or ulcerations. Additional medical history includes hypertension for which she has been prescribed hydrochlorothiazide at $25 \mathrm{mg}$ per day and ramipril at $10 \mathrm{mg}$ per day. She also takes aspirin at $81 \mathrm{mg}$ per day. On physical examination, she weighs $165 \mathrm{lbs}$, waist circumference is 30 inches, height is 68 inches, blood pressure sitting is $130 / 85$ and her pulse examination reveals a normal femoral and absent popliteal and pedal pulses on the right. On the left, her pulse examination reveals a normal femoral, an absent popliteal, and absent pedal pulses. Her resting ankle-brachial index is 0.70 for the left lower extremity and 0.74 for the right lower extremity. Her lipid profile reveals a total cholesterol of 225 $\mathrm{mg} / \mathrm{dL}$, and low-density lipoprotein of $160 \mathrm{mg} / \mathrm{dL}$, and a high-density lipoprotein of $36 \mathrm{mg} / \mathrm{dL}$. She has a normal glucose.

\section{Background}

Peripheral arterial disease (PAD) commonly refers to atherosclerosis of the abdominal aorta and arter- 
ies of the lower extremities. ${ }^{1}$ The disease affects men and women with a prevalence of $12 \%$ in the community and up to $26 \%$ in certain populations (ie, African American women with hypertension). ${ }^{2,3}$

Walking impairment is common among patients with PAD. The manifestations of walking impairment include reduced walking distance, walking speed, and stair climbing. Because of a compromise in walking, daily activity is reduced in these patients. The compromise in walking ability has been reported to be greater in women than men with PAD. ${ }^{4}$ Prior work evaluating differences in walking impairment by gender have not included cohorts that were similarly divided by race.

In addition to leg symptoms, patients with $\mathrm{PAD}$, either asymptomatic or symptomatic, are at increased risk for developing new coronary events and death from cardiovascular disease. For patients with an ankle-brachial index (ABI) of less than 0.85 , the relative risk (RR) of mortality at 10 years has been found to be 2.4 [ $95 \%$ confidence interval (CI) $1.60,3.48] .{ }^{5}$ Criqui et $\mathrm{al}^{6}$ demonstrated that within a cohort of patients with PAD whose mean age was 66 years, the RR for coronary heart disease (CHD) and cardiovascular disease (CVD) mortality for patients, without evidence of CVD at baseline, was 4.3 and 6.3, respectively. Because patients with $\mathrm{PAD}$ are at increased risk for all-cause mortality and cardiovascular morbidity as well as mortality, a lack of appreciation of the severity of this disease by gender could lead to inadequate process of care and inadequate risk reduction for cardiovascular events for vulnerable groups, particularly minority women.

In this article, we present our findings on the burden of PAD in women and men from 3 racial groups [white, African American, and Hispanic (both English and Spanish speaking)] who were screened for PAD.

\section{Methods}

\section{Recruitment Strategies}

The Baylor College of Medicine Institutional Review Board approved this study. We screened patients from the Michael E. DeBakey Veterans Affairs Medical Center and 3 primary care clinics within the Harris County Hospital District, from September 2000 through August 2001. Details of our recruitment of consecutive patients receiving primary care have been published previously. ${ }^{7}$

\section{Measurement of the ABI}

PAD was defined by an ABI below 0.9. A $5 \mathrm{mHz}$ hand-held Doppler with an attached stethoscope was used to measure systolic blood pressures at rest in both brachial arteries and in the dorsalis pedis and posterior tibial arteries. The ABI was calculated based on obtaining an initial reading followed by a second reading of the initially higher reading from the dorsalis pedis and posterior tibial artery systolic blood pressures. ${ }^{8}$ The average of the 2 readings was used as the ankle systolic pressure for that leg. The brachial systolic pressure was the average of the systolic blood pressures for each arm. If there was more than a $10 \mathrm{~mm} \mathrm{Hg}$ difference between the brachial systolic blood pressures in each arm, the higher arm was measured twice; the average of these 2 readings was the brachial pressure used to calculate the ABI for each leg. If the $\mathrm{ABI}$ of either leg was less than 0.9 , the patient was defined as having disease. All patients, with or without $\mathrm{PAD}$, completed all questionnaires.

\section{Measurement of Leg Symptoms and Comorbidities}

Each of the 4 questionnaires provided additional information about our cohort. The San Diego Claudication Questionnaire (SDCQ), ${ }^{9}$ a validated 9-item questionnaire that can be completed within 5 minutes, was used to detect claudication. The SDCQ specifically asks whether the symptoms are present in the right, left, or both legs. For our study, we placed patients in 3 symptom categories: no pain, atypical leg pain (ie, pain at rest, non-calf exercise leg pain, and "non-Rose" exercise calf pain), or Rose claudication. The patient's leg symptoms were categorized based on the most severely symptomatic leg (claudication $>$ atypical leg symptoms $>$ asymptomatic disease).

In addition to leg symptoms, we obtained information on walking impairment using the validated Walking Impairment Questionnaire (WIQ). ${ }^{10}$ The WIQ is a brief and simple means of assessing patient-reported walking ability and was developed for use in large epidemiologic studies to replace the more time-consuming exercise treadmill testing; it usually requires approximately 5 minutes for completion. The WIQ estimates walking distance, walking speed, and stair-climbing capacity in the community.

For walking distance, the questionnaire ascertains the patient's degree of difficulty in walking specific distances, incrementally increasing in se- 
verity (eg, walking indoors or 20 feet; walking less than half a block or 50 feet). The degree of difficulty is ranked on a 0 to 4 Likert scale in which 0 represents the inability to walk a specific distance and 4 represents no difficulty walking a specific distance.

For both walking speed and stair climbing, the same 0 to 4 Likert scale is used. For walking speed, the patient answers 4 questions in reference to the degree of difficulty in walking a certain speed: walking 1 block slowly, walking 1 block at an average speed, walking 1 block quickly, and running or jogging 1 block. For stair climbing, patients respond to 3 questions on the degree of difficulty of climbing 1 flight of stairs, 2 flights of stairs, and 3 flights of stairs. Scoring for each of the 3 components is based on the units of measurement described for each item (eg, 20 feet) and the patient's reported difficulty for each item-the less difficulty walking at a certain speed or climbing a certain number of stairs results in a higher score.

To determine health-related quality of life, we used the previously validated Medical Outcomes Study Short Form (SF-36). The SF-36 contains 36 items, which measure 8 health concepts. ${ }^{11}$ The 8 health concepts (ie, scales) are physical function, role limitations (physical problems), pain, social functioning, mental health, role limitations (emotional problems), vitality, and general health perceptions. The SF-36 scales are scored using the Likert method of summated ratings. ${ }^{12,14}$

We ascertained past medical history of cardiovascular disease, atherosclerotic risk factors, process of care (eg, medication use), and sociodemographics using the Lifestyle and Clinical Survey (LCS). This is an interviewer-administered questionnaire that requires less than 10 minutes to complete. ${ }^{15}$

Because we screened both English- and Spanishspeaking patients, each questionnaire (ie, SDCQ, WIQ, LCS) was either translated into Spanish by our team of bilingual research assistants ${ }^{16}$ and investigators or previously translated by an outside service (SF-36). The translation process involved one forward and 2 backward translations by bilingual interpreters who then decided, during 3 separate meetings, on a final Spanish version for each of the questionnaires.

\section{Statistical Analysis}

Differences between groups (men versus women or patients with and without $\mathrm{PAD}$ ) on continuous variables were tested using a $t$ test, with $P$ values less than .05 considered significant. Differences on categorical variables were assessed using the $\chi^{2}$ test for independence.

Univariate odds ratios (OR) with 95\% CI were calculated for each of the demographic characteristics and comorbidities of interest. In addition, we ran 3 logistic regression models to obtain the adjusted odds of having PAD for each of the demographic and comorbidity variables, accounting for other covariates. The full model contained gender, education, marital status, income, current work status, race, age, body mass index (BMI), whether the patient was on antiplatelet therapy, whether the patient was on blood pressure medication, current smoking status, and whether the patient had the following conditions: arthritis, high cholesterol, diabetes, hypertension, myocardial infarction, and stroke. The second model was a subset of the full model, consisting of gender, comorbidities, and sociodemographics. The third model was built using a stepwise procedure, in which variables entered the model if the $P$ value was .20 or less and remained in the model if the $P$ value was .05 or less. This model allowed us to identify the predictors most highly associated with PAD in our study sample.

\section{Results}

We approached a total of 457 patients, and 403 patients $(88.2 \%)$ were enrolled. Within our cohort, we enrolled 151 patients from the Michael E. DeBakey Veterans Affairs Medical Center, and the remainder of the patients from 3 sites within the Harris County Hospital district. Of the 403 patients enrolled, 208 (51.6\%) were women and 195 (48.4\%) were men. The population was similarly divided by race, with 136 whites, 136 African Americans, and 131 Hispanics. Eighty-one (61.8\%) of the Hispanic patients were Spanish speaking (predominant language spoken at home). Of the 81 Spanish-speaking Hispanic patients, 30 were male and 51 were female. The mean age of the cohort was $63.8 \pm 0.4$ years and the mean ABI was $1.06 \pm$ 0.01 (Table 1). The prevalence of diabetes mellitus was $35.6 \%$ for women and $40.5 \%$ for men $(P=$ .30). Among the entire cohort, current smoking was more prevalent in men than women $(24.1 \%$ vs $13.9 \% ; P=.009)$.

Among the patients who screened positive for disease, the mean $\mathrm{ABI}$ was $0.72 \pm 0.02(0.71 \pm 0.03$ 


\begin{tabular}{|c|c|c|c|c|}
\hline Demographics and Comorbidities & $\begin{array}{l}\text { Total Cohort } \\
403(100 \%)\end{array}$ & $\begin{array}{c}\text { Women } \\
208(51.6 \%)\end{array}$ & $\begin{array}{c}\text { Men } \\
195(48.4 \%)\end{array}$ & $P$ Value \\
\hline Age, mean $\pm \mathrm{SE}$ & $63.8 \pm 0.36$ & $63.4 \pm 0.48$ & $64.2 \pm 0.55$ & .33 \\
\hline Ankle-brachial index, mean $\pm \mathrm{SE}$ & $1.06 \pm 0.01$ & $1.04 \pm 0.01$ & $1.08 \pm 0.02$ & .08 \\
\hline Body mass index, mean $\pm \mathrm{SE}$ & $30.5 \pm 0.36$ & $31.6 \pm 0.63$ & $29.8 \pm 0.43$ & .02 \\
\hline $\begin{array}{l}\text { Education } \\
<8 \text { years } \\
8-11 \text { years } \\
\text { Graduated from high school } \\
\text { Graduated from college }\end{array}$ & $\begin{array}{r}106(26.3 \%) \\
85(21.1 \%) \\
169(41.9 \%) \\
42(10.4 \%)\end{array}$ & $\begin{array}{l}61(29.3 \%) \\
49(23.6 \%) \\
84(40.4 \%) \\
14(6.7 \%)\end{array}$ & $\begin{array}{l}45(23.1 \%) \\
36(18.5 \%) \\
85(43.6 \%) \\
28(14.4 \%)\end{array}$ & .03 \\
\hline Diabetes mellitus & $153(37.9 \%)$ & $74(35.6 \%)$ & $79(40.5 \%)$ & .30 \\
\hline Current smoking & $76(18.9 \%)$ & $29(13.9 \%)$ & $47(24.1 \%)$ & .009 \\
\hline Current use of blood pressure medication & $267(66.2 \%)$ & $128(61.5 \%)$ & $139(71.3 \%)$ & .04 \\
\hline Disease Prevalence by Gender and Race & $\begin{array}{l}\text { Total Cohort } \\
\mathrm{N}=403\end{array}$ & $\begin{array}{c}\mathrm{PAD} \\
\mathrm{N}=67\end{array}$ & $\begin{array}{l}\text { Non-PAD } \\
N=336\end{array}$ & $P$ Value \\
\hline $\begin{array}{l}\text { Women } \\
\text { White } \\
\text { African American } \\
\text { Hispanic* }\end{array}$ & $\begin{array}{l}55(13.6 \%) \\
82(20.3 \%) \\
71(17.6 \%)\end{array}$ & $\begin{array}{c}5(7.5 \%) \\
18(26.9 \%) \\
10(14.9 \%)\end{array}$ & $\begin{array}{l}50(14.9 \%) \\
64(19.0 \%) \\
61(18.1 \%)\end{array}$ & .11 \\
\hline $\begin{array}{l}\text { Men } \\
\text { White } \\
\text { African American } \\
\text { Hispanic }\end{array}$ & $\begin{array}{l}81(20.1 \%) \\
54(13.4 \%) \\
60(14.9 \%)\end{array}$ & $\begin{array}{r}13(19.4 \%) \\
13(19.4 \%) \\
8(11.9 \%)\end{array}$ & $\begin{array}{l}68(20.2 \%) \\
41(12.2 \%) \\
52(15.5 \%)\end{array}$ & .29 \\
\hline
\end{tabular}

${ }^{*}$ Hispanic includes both English and Spanish speaking

for women; $0.71 \pm 0.03$ for men; $P=.92$ ). In our sample, the prevalence of PAD in women was $15.9 \%$ compared with $17.4 \%$ in men. By gender and among patients with PAD, there was no difference in the prevalence of several atherosclerotic risk factors most notably diabetes mellitus, current smoking, use of blood pressure medication, or hypercholesterolemia (Table 2).
Leg symptoms, particularly atypical leg symptoms, were common among men and women with and without PAD (Table 3). When compared with women without disease, women with PAD had poorer walking distance and walking speed. In contrast, walking impairment was not statistically different when comparing men with and without $\mathrm{PAD}$. Intermittent claudication was present in

Table 2. Characteristics of Patients with PAD

\begin{tabular}{|c|c|c|c|}
\hline & \multicolumn{2}{|c|}{$\mathrm{N}(\%)$} & \multirow[b]{2}{*}{$P$ Value } \\
\hline & $\begin{array}{l}\text { Women } \\
(\mathrm{n}=33)\end{array}$ & $\begin{array}{c}\text { Men } \\
(\mathrm{n}=34)\end{array}$ & \\
\hline Diabetes mellitus & $19(57.6)$ & $18(52.9)$ & $>.20$ \\
\hline Current smoking & $9(27.3)$ & $11(32.3)$ & $>.20$ \\
\hline Current use of blood pressure medication & $29(87.9)$ & $27(79.4)$ & $>.20$ \\
\hline Hypercholesterolemia & $17(51.5)$ & $13(38.2)$ & $>.20$ \\
\hline Congestive heart failure & $2(6.1)$ & $3(8.8)$ & $>.20$ \\
\hline Atrial fibrillation & $7(21.2)$ & $4(11.8)$ & $>.20$ \\
\hline Rheumatoid arthritis or any arthritis other than rheumatoid & $23(69.7)$ & $18(52.9)$ & .16 \\
\hline Stroke or transient ischemic attack & $4(12.1)$ & $3(8.8)$ & $>.20$ \\
\hline $\begin{array}{l}\text { Use of at least one nonsteroidal anti-inflammatory agent } \\
\text { daily, weekly, or occasionally* }\end{array}$ & $16(48.5)$ & $24(70.6)$ & .06 \\
\hline Use of pentoxifylline (Trental) & $1(3.0)$ & $0(0)$ & $>.20$ \\
\hline Use of any antiplatelet agent $\dagger$ & $11(33.3)$ & $15(44.1)$ & $>.20$ \\
\hline
\end{tabular}

* Nonsteroidal anti-inflammatory agents included the following: Ecotrin, Bayer, ibuprofen, Motrin, Aleve, Advil, BC (a powder substance used for pain relief; its ingredients are salicylate, caffeine, and salicylamide), celecoxib, rofecoxib, naproxen, and Alka Seltzer. † Antiplatelet agents included aspirin, ticlopidine, and clopidogrel. 


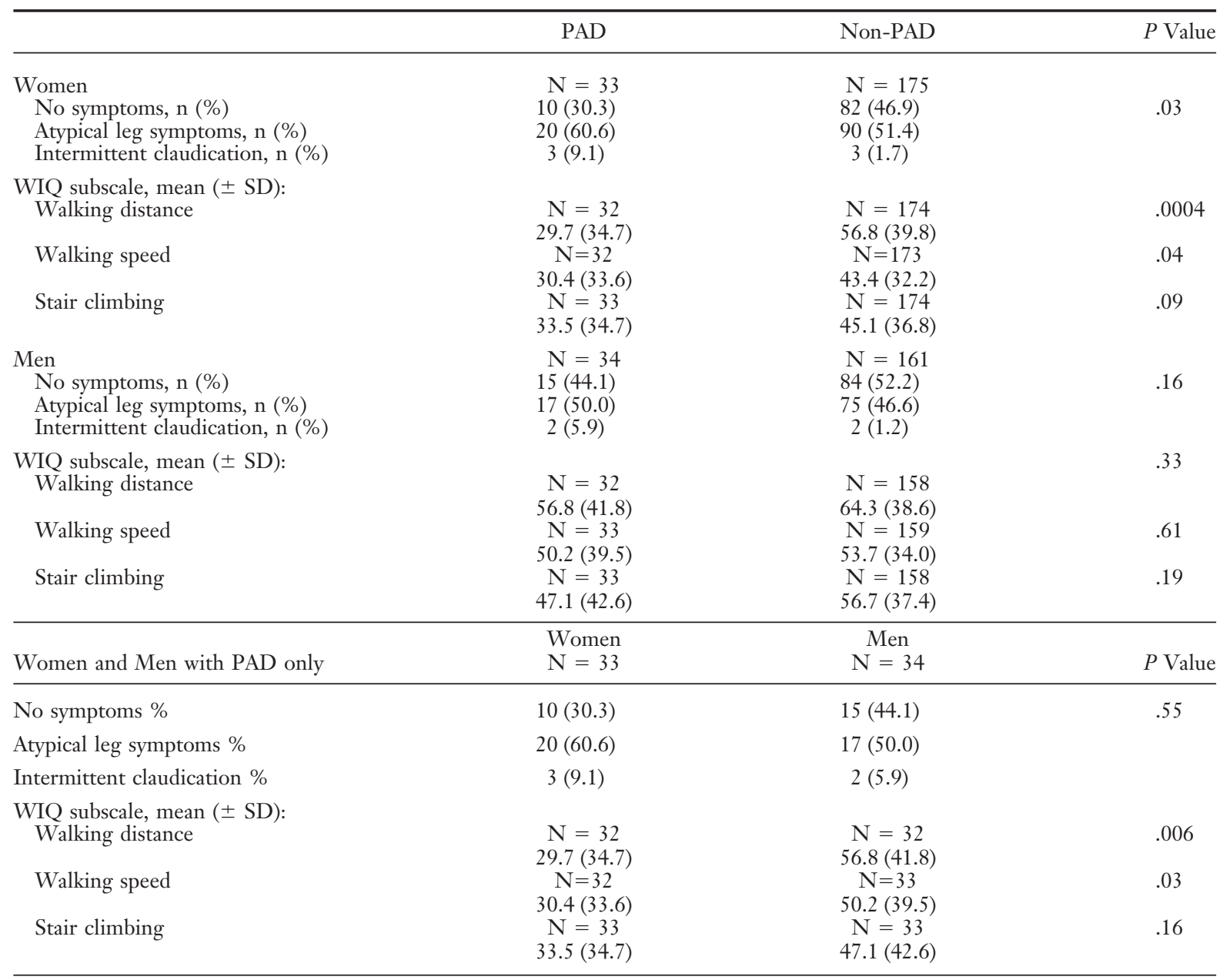

$9.1 \%$ of women and $5.9 \%$ of men who screened positive for disease. Among patients who screened positive for disease, comparing genders, mean walking distance was $29.7 \pm 34.7$ for women and $56.8 \pm 41.8$ for men $(P=.006)$.

Female gender and race were not statistically significant risk factors for PAD. In our stepwise regression model, statistically significant risk factors for PAD were work status of not currently employed, diabetes mellitus, use of blood pressure medication, current smoking status, and BMI (Table 4). Diabetes mellitus demonstrated the greatest association with a diagnosis of PAD (OR 3.42; $95 \%$ CI 1.68, 6.95). In looking at each gender separately (eg, a model involving only women in the cohort), the risk factors for PAD were diabetes mellitus, current smoking, and use of blood pressure medication; these risk factors were the same for a model involving only men. Within these additional analyses, the risk factor demonstrating the highest as- sociation for a diagnosis of PAD among both women and men was the use of blood pressure medication (OR 7.69; 95\% CI 1.5, 39.2 for women; OR 2.76; 95\% CI 1.4, 5.5 for men).

Health-related quality of life scores as measured by the SF-36 (Table 5) were similar between women and men in the cohort. Among patients with $\mathrm{PAD}$, physical functioning was significantly lower for women $(37.5 \pm 26.4)$ when compared with men $(52.4 \pm 32.5), P=.04$. Also in patients with $\mathrm{PAD}$, general health was lower for women $(43.8 \pm 22.4)$ when compared with men (55.1 \pm 23.4), $P=.05$.

\section{Discussion}

Within our cohort, we found similarities and differences between men and women who were screened for PAD. First, PAD is a common diagnosis for women and men who receive medical treatment in a primary care setting. African Amer- 
Table 4. Predictors of Peripheral Arterial Disease

\begin{tabular}{|c|c|c|c|c|c|c|c|}
\hline \multirow[b]{2}{*}{ Variable } & \multirow{2}{*}{$\begin{array}{c}\text { Univariate } \\
\text { OR }(95 \% \mathrm{CI})\end{array}$} & \multicolumn{2}{|c|}{ Full Model* } & \multicolumn{2}{|c|}{ Subset of Full Model $\dagger$} & \multicolumn{2}{|c|}{ Reduced Model $\neq$} \\
\hline & & OR (95\% CI) & $P$ Value & OR $(95 \% \mathrm{CI})$ & $P$ Value & OR (95\% CI) & $P$ Value \\
\hline Gender (women/men) & $1.12(0.66,1.89)$ & $0.70(0.34,1.44)$ & .33 & $0.89(0.49,1.59)$ & .69 & & \\
\hline $\begin{array}{c}\text { Education }(<\text { High School/ } \\
\geq \text { High School })\end{array}$ & $0.76(0.45,1.29)$ & $0.49(0.21,1.13)$ & .09 & & & & \\
\hline Currently married (y/n) & $0.78(0.46,1.32)$ & $1.06(0.51,2.24)$ & .87 & & & & \\
\hline Income $(<\$ 5000 / \geq \$ 5000)$ & $1.23(0.61,2.48)$ & $0.94(0.39,2.27)$ & .89 & & & & \\
\hline Current work status (n/y) & $2.06(1.00,4.25)$ & $2.52(0.92,6.92)$ & .07 & & & $2.67(1.04,6.86)$ & .04 \\
\hline Antiplatelet (y/n) & $1.36(0.79,2.33)$ & $1.00(0.47,2.15)$ & .99 & $1.00(0.55,1.83)$ & .99 & & \\
\hline Arthritis (y/n) & $1.16(0.69,1.97)$ & $1.13(0.54,2.36)$ & .74 & $1.09(0.61,1.95)$ & .77 & & \\
\hline High cholesterol (y/n) & $0.97(0.57,1.64)$ & $0.90(0.44,1.84)$ & .77 & $0.89(0.50,1.57)$ & .68 & & \\
\hline Diabetes (y/n) & $2.34(1.37,3.98)$ & $3.52(1.67,7.41)$ & .0009 & $2.64(1.47,4.73)$ & .001 & $3.42(1.68,6.95)$ & .0007 \\
\hline Hypertension (y/n) & $2.32(1.20,4.51)$ & $1.27(0.26,6.12)$ & .77 & $0.64(0.19,2.22)$ & .48 & & \\
\hline MI (y/n) & $1.59(0.76,3.30)$ & $1.35(0.51,3.55)$ & .55 & $1.36(0.60,3.05)$ & .46 & & \\
\hline Stroke $(y / n)$ & $1.98(0.78,4.83)$ & $0.80(0.25,2.61)$ & .71 & $1.64(0.62,4.37)$ & .32 & & \\
\hline BP medication $(\mathrm{y} / \mathrm{n})$ & $3.02(1.52,5.97)$ & $2.36(0.54,10.42)$ & .26 & $3.30(0.94,11.51)$ & .06 & $2.69(1.14,6.37)$ & .02 \\
\hline Current smoker (y/n) & $2.13(1.17,3.86)$ & $2.63(1.17,5.89)$ & .02 & $2.83(1.45,5.55)$ & .002 & $2.37(1.11,5.06)$ & .03 \\
\hline Ever smoker (y/n) & $1.77(1.01,3.09)$ & & & & & & \\
\hline $\begin{array}{l}\text { Race } \\
\text { black/white } \\
\text { Latino/white }\end{array}$ & & $\begin{array}{l}1.59(0.67,3.79) \\
1.85(0.62,5.55)\end{array}$ & $\begin{array}{l}.30 \\
.27\end{array}$ & $\begin{array}{l}1.57(0.79,3.15) \\
1.01(0.47,2.20)\end{array}$ & $\begin{array}{l}.20 \\
.98\end{array}$ & & \\
\hline Age (continuous) & & $1.03(0.98,1.08)$ & .25 & $1.03(0.99,1.07)$ & .12 & & \\
\hline BMI (continuous) & & $0.93(0.88,1.00)$ & .04 & & & $0.94(0.88,0.99)$ & .03 \\
\hline
\end{tabular}

* Full model contains the following variables: gender, education, married, income, current work status, antiplatelet, arthritis, high cholesterol, diabetes, hypertension, myocardial infarction (MI), stroke, blood pressure (BP) medication, current smoker, race (using dummy variables with white as referent), age, and body mass index (BMI). There are missing data for BMI (49 observations), work status (56 observations), and income (24 observations)

† Subset of full model contains the following variables: antiplatelet, arthritis, high cholesterol, diabetes, hypertension, MI, stroke, BP medication, current smoker, race (using dummy variables with white as referent), and age.

$\ddagger$ Reduced model is built using stepwise procedure with variables having $P \leq .20$ entering the model and those with $P \leq .05$ remaining in the model. Order of variables entered: diabetes, current smoking, age, current work, age removed, BP medication entered, BMI entered, education entered, and then education removed.

ican and Hispanic women have a higher, although not statistically significant, prevalence of disease when compared with white women. Second, walking impairment is greater among women with PAD when compared with women without disease and to men with disease. Third, the risk factors for PAD are the same for women and men. Finally, healthrelated quality of life is compromised to a greater extent in women with PAD when compared with men with PAD.

Our finding of a similar prevalence of PAD between men and women has been demonstrated in prior work. ${ }^{4}$ We have added to this body of literature by screening for disease in a racially diverse population that included Spanish-speaking patients-an often under-represented group within clinical research. Within our cohort, men and women predominantly 55 years of age and older, there was a high prevalence of atherosclerotic risk factors. Our findings, along with findings from other PAD prevalence studies ${ }^{2,8,17}$ support the importance of screening for PAD in both men and women at least 50 years of age with risk factors of diabetes mellitus or current smoking.

In contrast to the prevalence of disease, walking impairment did differ by gender. Within our cohort, women with PAD had significantly lower mean values for walking distance and walking speed when compared with men with PAD. The magnitude of the differences in walking distance and speed for women and men without PAD was relatively low (approximately 8 to 10 percentage points of difference). In contrast, there was a much greater difference in both walking distance and speed between women and men with PAD (approximately 20 to 27 percentage points of difference). Although 
Table 5. Comparisons by Gender and PAD Status of SF-36 Quality of Life Scores

\begin{tabular}{|c|c|c|c|c|c|c|c|c|c|}
\hline & \multicolumn{3}{|c|}{ Entire Cohort $(n=403)$} & \multicolumn{3}{|c|}{ PAD only $(\mathrm{n}=67)$} & \multicolumn{3}{|c|}{ no PAD only $(\mathrm{n}=336)$} \\
\hline & $\begin{array}{c}\text { Female } \\
\mathrm{N}=208 \\
\text { mean }(\mathrm{SD})\end{array}$ & $\begin{array}{c}\text { Male } \\
\mathrm{N}=195 \\
\text { mean }(\mathrm{SD})\end{array}$ & $\begin{array}{c}P \\
\text { Value }\end{array}$ & $\begin{array}{c}\text { Female } \\
\mathrm{N}=33 \\
\text { mean }(\mathrm{SD})\end{array}$ & $\begin{array}{c}\text { Male } \\
\mathrm{N}=34 \\
\text { mean }(\mathrm{SD})\end{array}$ & $\begin{array}{c}P \\
\text { Value }\end{array}$ & $\begin{array}{c}\text { Female } \\
\mathrm{N}=175 \\
\text { mean }(\mathrm{SD})\end{array}$ & $\begin{array}{c}\text { Male } \\
\mathrm{N}=161 \\
\text { mean }(\mathrm{SD})\end{array}$ & $P$ Value \\
\hline \multicolumn{10}{|l|}{8 Subgroup Scores: } \\
\hline Physical functioning & $52.8(28.1)$ & $57.3(31.9)$ & $一^{*}$ & $37.5(26.4)$ & $52.4(32.5)$ & .04 & $55.7(27.9)$ & $58.3(31.8)$ & $>.20$ \\
\hline Role physical index & $41.3(42.1)$ & $39.4(41.6)$ & $>.20$ & $20.7(34.0)$ & $31.6(41.9)$ & $>.20$ & $45.1(42.4)$ & $41.1(41.5)$ & $>.20$ \\
\hline Bodily pain & $54.4(27.7)$ & $53.3(29.6)$ & $>.20$ & $44.7(26.8)$ & $57.0(28.4)$ & - $^{*}$ & $56.3(27.6)$ & $52.5(29.9)$ & $>.20$ \\
\hline General health & $54.1(23.2)$ & $50.0(21.2)$ & -* & $43.8(22.4)$ & $55.1(23.4)$ & .05 & $56.1(22.9)$ & $49.0(20.6)$ & .003 \\
\hline Vitality & $50.5(25.9)$ & $48.5(26.9)$ & $>.20$ & $44.7(22.6)$ & $54.0(25.4)$ & 一 $^{*}$ & $51.6(26.4)$ & $47.3(27.2)$ & 一 $^{*}$ \\
\hline Social functioning index & $71.3(27.2)$ & $67.5(30.3)$ & - $^{*}$ & $64.0(25.5)$ & $72.4(27.7)$ & 一 $^{*}$ & $72.6(27.3)$ & $66.5(30.8)$ & .05 \\
\hline Role emotional index & $58.6(43.3)$ & $62.6(41.9)$ & $>.20$ & $49.5(42.6)$ & $67.6(42.2)$ & - $^{*}$ & $60.3(43.3)$ & $61.5(41.9)$ & $>.20$ \\
\hline Mental health index & $70.3(22.1)$ & $70.8(23.7)$ & $>.20$ & $70.9(22.1)$ & $78.6(21.4)$ & - $^{*}$ & $70.2(22.1)$ & $69.1(23.9)$ & $>.20$ \\
\hline \multicolumn{10}{|l|}{2 Summary Measures: } \\
\hline Physical component score & $36.6(11.2)$ & $36.4(11.7)$ & $>.20$ & $29.5(8.8)$ & $34.6(11.4)$ & .05 & $37.9(11.2)$ & $36.7(11.8)$ & $>.20$ \\
\hline Mental component score & $48.8(12.5)$ & $48.4(12.5)$ & $>.20$ & $49.0(12.5)$ & $53.0(11.7)$ & —* & $48.8(12.6)$ & $47.5(12.5)$ & $>.20$ \\
\hline
\end{tabular}

${ }^{*} P>.05<.20$.

the presence of greater muscle mass and body height for men in general would seem to explain the differences in walking ability between men and women without disease, the significant increase in the differences in their walking parameters in the presence of $\mathrm{PAD}$ - a greater burden of walking impairment among women versus men with PAD—is of concern. One prior study demonstrated that gender variation in walking impairment might be partially explained by variation in leg strength with women having poorer leg strength than men. ${ }^{4}$ Although we did not capture leg strength within this study, women within the cohort had a higher mean BMI when compared with men. Because a BMI that is increased secondary to a higher proportion of non-lean versus lean tissue often correlates with physical inactivity, ${ }^{18}$ this higher mean $\mathrm{BMI}$ in women may be a marker for poorer leg strength.

Aside from variation in walking impairment by gender, we did not find that gender was a significant factor in the multivariate model. Risk factors for PAD were the same for both women and men; for both sexes, the risk factor with the greatest association with $\mathrm{PAD}$ was hypertension requiring the use of medication.

Although there was a trend toward a higher prevalence of disease in African American women compared with white women, there was no statistically significant association of race with a diagnosis of PAD within the multivariate model. In re- cently published work, ${ }^{19}$ we found that the increased risk for PAD among African American patients was attenuated after adjusting for education. As our study may not have had enough power to detect a statistically significant difference in the prevalence of disease by race, a larger racially diverse cohort of patients with $\mathrm{PAD}$ is needed to address the role of race and gender as risk factors for PAD and to understand the role of other social factors as confounders for the association of race/ gender with this chronic illness.

Not surprisingly, chronic disease is a risk for poor quality of life. ${ }^{20}$ In assessing health-related quality of life among members of our cohort with $\mathrm{PAD}$, we found that women had lower scores for physical functioning and general health when compared with men. A lower quality of life among women with PAD is not surprising given the greater compromise in walking ability. The compromised quality of life for women with PAD has been reported in a prior study. ${ }^{21} \mathrm{~A}$ patient with $\mathrm{PAD}$ is at risk for poor walking and the inability to carry out activities of daily living including grocery shopping. In addition, personal relationships can be negatively impacted as walking through the mall with loved ones or playing with grandchildren becomes a great challenge. Interestingly, within our cohort among patients who did not screen positive for disease, men had lower quality of life scores when compared with women. This lower quality of life is probably a reflection of the burden of other 
conditions (eg, smoking, use of blood pressure medication) in men relative to women within our cohort.

One limitation of our study was the cross-sectional design. Because of this design, we are not able to determine the length of time that a patient had a given risk factor or the incidence of PAD. However, we have clearly defined the prevalence of disease by gender and the variation in walking impairment between the 2 sexes within this racially diverse population. An additional limitation is the use of self-reporting to determine a patient's risk factor profile. The limitation is probably greatest for patients with hypercholesterolemia. In prior work, we found that patient self-report when compared with chart reviews reliably captures many atherosclerotic risk factors. ${ }^{15}$ Finally, the power to detect statistically significant differences was also a limitation in our study. Our power was lowest in finding differences between the 33 women and the 34 men with PAD. We had slightly less than $70 \%$ power to detect medium-sized differences in the proportion of women with various comorbidities compared with men. In examining differences in the 3 categories of leg symptoms, power was $59 \%$. For the WIQ subscales, we had $80 \%$ power to detect a medium to large effect size of 0.62 . Our power to detect the fairly small effect size seen between women and men with PAD for stair climbing (effect size $=0.35$ ) was only $41 \%$. Whereas the power was somewhat low for detecting differences in the prevalence of leg symptom subtypes between men and women with $\mathrm{PAD}$, the power to detect differences in the continuous and categorical demographic and comorbidity variables between men and women in the overall cohort was more than $90 \% .^{22}$ We had more than $90 \%$ power to detect a medium size difference (medium effect size of 0.30 ) in the prevalence of leg symptoms between women with and without PAD and between men with and without PAD. In testing for differences between women with and without PAD and between men with and without $\mathrm{PAD}$ in walking distance, walking speed, and stair climbing, our power to detect a medium effect size was slightly over $80 \%$.

The case patient warrants risk factor modification including lipid control (goal of $<100 \mathrm{mg} / \mathrm{dL}$ ) and continued use of an antiplatelet agent because patients with $\mathrm{PAD}$ are at risk for a myocardial infarction or ischemic cerebrovascular accident. The use of cilostazol or supervised exercise tread- mill training (3 times per week for 6 months) is warranted for leg symptoms to improve her lower limb function.

In conclusion, $\mathrm{PAD}$ is a common illness among women, particularly minority women. The burden of walking impairment is greater for women than men with PAD. In addition, health-related quality of life, particularly physical functioning, is greatly compromised in women with PAD. Future studies are needed to assess methods-including screening protocols - that will help to maintain proper limb functioning in patients, particularly women from racially diverse backgrounds, who are diagnosed with PAD.

\section{References}

1. McDermott MM, Greenland P. Clinical significance and functional implications of peripheral arterial disease. In: Olin JW, editor. Clinical evaluation and office-based detection of peripheral arterial disease. An office-based approach to the diagnosis and treatment of peripheral arterial disease. Parts I-VIII. 1st edition. Manchester: Society for Vascular Medicine and Biology 1998; p. 20-6.

2. Newman A, Sutton-Tyrrell K, Kuller L. Lowerextremity arterial disease in older hypertensive adults. Arterioscler Thromb 1993;13:555-62.

3. Newman A. Peripheral arterial disease: insights from population studies of older adults. J Am Geriatr Soc 2000;48:1157-62.

4. McDermott M, Greenland P, Liu K, et al. Sex differences in peripheral arterial disease: leg symptoms and physical functioning. J Am Geriatr Soc 2003;51: 222-8.

5. McKenna M, Wolfson S, Kuller L. The ratio of ankle and arm arterial pressure as an independent predictor of mortality. Atherosclerosis 1991;87:11928.

6. Criqui M, Langer R, Fronek A, et al. Mortality over a period of 10 years in patients with peripheral arterial disease. N Engl J Med 1992;326:381-6.

7. Collins T, Petersen N, Suarez-Almazor M, Ashton C. The prevalence of peripheral arterial disease in a racially diverse population. Arch Intern Med 2003; 163:1469-74.

8. Hirsch A, Criqui M, Treat-Jacobson D, et al. Peripheral arterial disease detection, awareness, and treatment in primary care, JAMA 2001;286:1317-24.

9. Criqui M, Denenberg J, Bird C, Fronek A, Klauber $\mathrm{M}$, Langer R. The correlation between symptoms and non-invasive test results in patients referred for peripheral arterial disease testing. Vasc Med 1996;1: 65-71.

10. Regensteiner J, Steiner J, Panzer R, Hiatt W. Evaluation of walking impairment by questionnaire in 
patients with peripheral arterial disease. J Vasc Med Biol 1990;2:142-52.

11. Ware JE, Snow KK, Kosinski M, Gandek B. SF-36 Health Survey: manual and interpretation guide. Lincoln (RI):2000; Quality Metric Incorporated.

12. Edwards AL. Techniques of attitude scale construction. New York: Appleton-Century-Crofts, Inc. 1957.

13. Likert R. A technique for the measurement of attitudes. Arch Psychol 1932;140:5-55.

14. Ware JE, Gandek B. Methods for testing data quality, scaling assumptions, and reliability: the IQOLA Project approach. J Clin Epidemiol 1998;51:945-52.

15. Collins T, O'Malley K, Petersen N, Suarez-Almazor M. The Lifestyle and Clinical Survey: a pilot study to validate a medical history questionnaire. Fed Pract 2005;22:25-46.

16. Collins TC, Suarez-Almazor M, Petersen NJ, O'Malley KJ. A. Spanish translation of the Walking Impairment Questionnaire was validated for patients with peripheral arterial disease. J Clin Epidemiol 2004:57;1305-15.
17. McDermott M, Kerwin D, Liu K, et al. Prevalence and significance of unrecognized lower extremity peripheral arterial disease in general medicine practice. J Gen Intern Med 2001;16:384-90.

18. Weinstein A, Sesso H, Lee IM, et al. Relationship of physical activity versus body mass index with type 2 diabetes in women. JAMA 2004;292:1188-94.

19. Collins T, Petersen N, Suarez-Almazor M, Ashton C. Ethnicity and peripheral arterial disease. Mayo Clin Proc 2005;80:48-54.

20. Mitra M, Chung M, Wilber N, Klein W. Smoking status and quality of life a longitudinal study among adults with disabilities. Am J Prev Med 2004;27:25860 .

21. Bloemenkamp D, Mali W, Tanis B, et al. Functional health and well being of relatively young women with peripheral arterial disease is decreased but stable after diagnosis. J Vasc Surg 2003;38:104-10.

22. Cohen J. Statistical power analysis for the behavioral sciences. Hillsdale (NJ): Lawrence Erlbaum Associates, 1988. 